\title{
NON-SIMULTANEOUS BLOW-UP FOR A SEMILINEAR PARABOLIC SYSTEM WITH LOCALIZED REACTION TERMS
}

\author{
ATSUKO OKADA
}

Abstract. In this paper, we study positive blow-up solutions of the semilinear parabolic system with localized reactions $u_{t}=\Delta u+v^{r}+u^{p}(0, t), v_{t}=\Delta v+u^{s}+v^{q}(0, t)$ in the ball $B=\{x \in$ $\left.\mathbb{R}^{N}:|x|<R\right\}$, under the homogeneous Dirichlet boundary condition. It is shown that nonsimultaneous blow-up may occur according to the value of $p, q, r$, and $s(p, q, r, s>1)$. We also investigate blow-up rates of all total blow-up solutions when simultaneous blow-up occurs.

Mathematics subject classification (2010): 34K51, 35B44.

Keywords and phrases: blow-up, parabolic system, simultaneous blow-up, non-simultaneous blow-up, total blow-up.

\section{REFERENCES}

[1] J. Bebernes, A. BRess An And A. LACEy, Total blow-up versus single point blow-up, J. Diff. Eqs., 73, (1988), 30-44.

[2] J. M. Chadam, A. Pierce And H-M. Yin, The blowup property of solutions to some diffusion equations with localized nonlinear reactions, J. Math. Anal. Appl., 169, (1992), 313-328.

[3] M. EsCOBEDO AND M. A. HERRERO, Boundedness and blow up for a semilinear reaction-diffusion system, J. Diff. Eqs., 89, (1991), 176-202.

[4] A. OKADA AND I. FUKUDA, Blow-up of solutions of nonlinear parabolic equations with localized reactions, GAKUTO International Ser. Math. Sci. Appl., 14, (2000), 358-366.

[5] A. OKADA AND I. FUKUDA, Total versus single point blow-up of solutions of a semilinear parabolic equation with localized reaction, J. Math. Anal. Appl., 281, (2003), 485-500.

[6] M. ÔTANI, $L^{\infty}$-Energy method, basic tools and usage, Progress in Nonlinear Differential Equations and Their Applications, 75, (2007), 357-376.

[7] J. D. RosSI AND P. SOUPLET, Coexistence of simultaneous and non-simultaneous blow-up in a semilinear parabolic system, Diff. Int. Eqs., 18, (2005), 405-418.

[8] P. Souplet, Uniform blow-up profiles and boundary behavior for diffusion equations with nonlocal nonlinear source, J. Diff. Eqs., 153, (1999), 374-406.

[9] P. Souplet, Single-point blow-up for a semilinear parabolic system, J. Eur. Math. Soc., 11, (2009), 169-188.

[10] P. Souplet And S. TAYAChI, Optimal condition for non-simultaneous blow-up in a reactiondiffusion system, J. Math. Soc. Japan, 56, 2 (2004), 571-584.

[11] S. ZHENG AND J. WANG, Total versus single point blow-up in heat equation with coupled localized sources, Asymptotic Analysis, 51, (2007), 133-156.

[12] J. WANG AND S. ZHeng, Total versus single point blow-up in a localized heat system, Front. Math. China, 5, 2 (2010), 341-359. 